\title{
HIGH PRECISION TOPOGRAPHIC MAPPING AT CHANG'E-3 LANDING SITE WITH MULTI-SOURCE DATA
}

\author{
Y. Liu ${ }^{\text {a }}$, B. Liu ${ }^{\text {a }}$, B. Xu ${ }^{\text {a }}$ Z. Liu ${ }^{\text {a }}$ K. Di ${ }^{\text {a, } *, \text { J. Zhou }}{ }^{\text {b }}$ \\ ${ }^{\text {a }}$ State Key Laboratory of Remote Sensing Science, Institute of Remote Sensing and Digital Earth, Chinese Academy of \\ Sciences, P.O.Box 9718, Datun Road, Chaoyang District, Beijing 100101, China - dikc@radi.ac.cn \\ ${ }^{\mathrm{b}}$ Beijing Aerospace Control Center, Beijing 100094, China
}

\section{Commission VI, WG IV/8}

KEY WORDS: Planetary Mapping, Multiresolution, Bundle, DEM/DTM, Orthorectification

\begin{abstract}
:
Chang'e-3 (CE-3) is the first lander and rover of China following the success of Chang'e-1 and Chang'e-2 (CE-2) orbiters. High precision topographic mapping can provide detailed terrain information to ensure the safety of the rover as well as to support scientific investigations. In this research, multi-source data are co-registered into a uniform geographic framework for high precision topographic mapping at the CE-3 landing site. CE-2 CCD images with $7 \mathrm{~m}$ - and $1.5 \mathrm{~m}$ - resolutions are registered using selfcalibration bundle adjustment method with ground control points (GCPs) selected from LRO WAC mosaic map and LOLA DTM. The trajectory of CE-3 descent images are recovered using self-calibration free net bundle adjustment, and then the topographic data is rectified by absolute orientation with GCPs selected from the adjusted CE-2 DEM and DOM. Finally, these topographic data are integrated into the same geographic framework for unified, multi-scale, high precision mapping of the CE-3 landing site. Key technologies and the mapping products of this research have been used to support the surface operations of CE-3 mission.
\end{abstract}

\section{INTRODUCTION}

The Chinese Lunar Exploration Program (CLEP, also called Chang'e program) was started since 2004. So far, China has successfully launched Chang'e-1 (CE-1) orbiter, Chang'e-2 (CE-2) orbiter and Chang'e-3 (CE-3) lander on 2007, 2010 and 2013, respectively. The orbiter cameras on board CE-1 and CE2 completed global mapping and local fine-scale mapping (Ouyang et al, 2010) and CE-3 realized China's first landing on the moon.

CE-2 orbiter carried a two line CCD camera which acquired 7 m-resolution images on $100 * 100 \mathrm{~km}$ circular orbit and $1.5 \mathrm{~m}$ resolution images at the perilune on $100 * 15 \mathrm{~km}$ elliptical orbit. Images with the former resolution have covered the whole lunar surface and 32 tracks with the later resolution were acquired at the CE-3 landing site-Mare Imbrium and Sinus Iridum (mostly but not completely covered). A global image map of the Moon with CE-2 $7 \mathrm{~m}$ - resolution was released by the CLEP on 6 February 2012 (Li, 2012). Wu et al (2014) performed a combined block adjustment of multiple tracks of CE-2 imagery and LOLA data.

CE-3 was launched on 2 December 2013, and landed on Mare Imbrium $\left(44.12^{\circ} \mathrm{N}, 19.51^{\circ} \mathrm{W}\right)$ on 14 December 2013 , and the rover Yutu was released to lunar surface and began its exploration soon after landing. During the landing phase, the descent camera carried by the lander acquired 4672 high and ultra-high resolution sequence images of the landing site. These descent images were used to monitor the landing process and pinpoint the locations of CE-3 lander and Yutu rover. This paper presents the high precision topographic mapping method and results using CE- 2 orbital images, CE- 3 descent images and control information from LRO data. The products have been used at Beijing Aerospace Control Center to support the surface operations of CE-3 mission.

\section{APPROACH}

The flow chart of the developed multi-source topographic mapping method is shown in Figure 1.

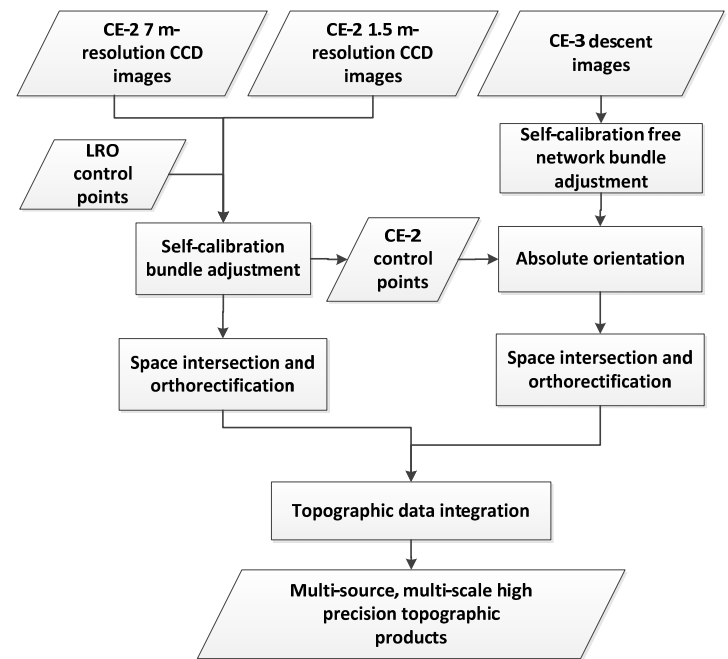

Figure 1. Flow chart of the high precision mapping method with multi-source data at CE-3 landing site

\footnotetext{
* Corresponding author.
} 
As the flow chart shows, geometric model of the $7 \mathrm{~m}$ - and 1.5 m-resolution CE-2 CCD images is refined using a selfcalibration bundle adjustment method (SCBA) with some control points (GCPs) selected from the LRO WAC DEM and DOM. The descending trajectory of CE-3 descent sequence images is also recovered after a self-calibration free network bundle adjustment; then absolute orientation of the descent images is performed with several GCPs selected from the adjusted CE-2 topographic products. Based on a series of matching strategy (SIFT-RANSAC-dense matching), sufficient points are accurately matched and their corresponding ground positions are calculated by space intersection. DEM and DOM are generated subsequently. Finally, the topographic data derived from multi-source data are integrated to build a unified, multi-scale, high precision map of the CE-3 landing site.

\section{GEOMETRIC PROCESSING OF CE-2 CCD IMAGES}

We have developed the rigorous geometric model of CE-2 CCD images according to its camera structure and exterior orientation process (Di et al., 2012), and then a self-calibration bundle adjustment (SCBA) method has been proposed for eliminating the inconsistencies in both image space and object space (Di et al., 2014). The interior orientation formula is refined by adding several calibration parameters as

$$
\begin{aligned}
& x^{\prime}=\left(x-x_{-} \text {offset }\right) / x_{-} \text {scale } \\
& y^{\prime}=\left(y-y_{-} \text {offset }\right) / y_{-} \text {scale }
\end{aligned}
$$

Exterior orientation parameters (EOPs) are fitted using $3^{\text {rd }}$ polynomials, and observation equations are built based on the modified collinearity equation as followed.

$$
\begin{aligned}
& x=-x_{-} \text {scale } \cdot f \cdot \bar{X} / \bar{Z}+x_{-} \text {offset } \\
& y=-y_{-} \text {scale } \cdot f \cdot \bar{Y} / \bar{Z}+y_{\text {_offset }}
\end{aligned}
$$

With some even-distributed tie points, bundle adjustment is performed to solve the calibration parameters, polynomial coefficients of EOPs and the ground points simultaneously. Several GCPs are selected from the LRO data and incorporated into the adjustment. Image coordinates of the GCPs are matched between CE-2 CCD images and LRO $100 \mathrm{~m}$ resolution WAC mosaic map (http://wms.lroc.asu.edu/lroc/view_rdr_product/WAC_GLOBA L_E300N3150_100M), and the horizontal coordinates (longitude and latitude) are obtained. The altitudes of the GCPs are derived from the LOLA DEM (http://ode.rsl.wustl.edu/moon/).

Two tracks of CE-2 CCD imagery covered the CE-3 landing site are chosen in this research, one is No.0570 track $(7 \mathrm{~m}$ resolution, 10,000 rows) acquired on 23 October, 2010 (UTC), the other is No.0236 track (1.5 m resolution, 15,000 rows) acquired on 27 October, 2010 (UTC). 270 tie points and 16 GCPs are selected to participate in the adjustment. After bundle adjustment, ground points by space intersection from No.0236 and No.0570 tracks respectively are combined to generate a DEM. For comparison purpose, another DEM is generated from the 3D points derived from the original image orientation parameters. Figure 2 shows the two DEMs and Tables 1 and 2 list the back-projection residual statistics before and after the adjustment. As
Figure 2 (a) and Table 1 show, DEM generated before adjustment has remarkable inconsistencies in both image space and object space. After adjustment, the back projection residuals reduced to sub pixel and the DEM tends to be consistent (shown in

Figure 2 (b) and Table 2), proving the effectiveness of the SCBA method.

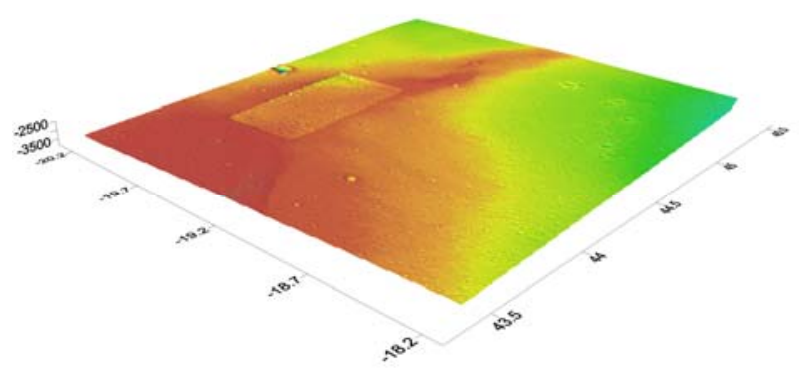

(a)

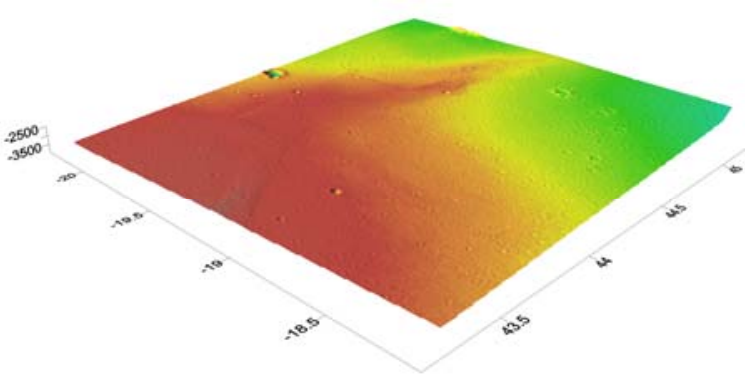

(b)

Figure 2. CE-2 DEMs generated (a) before and (b) after adjustment

Table 1.Back-projection residuals before adjustment

\begin{tabular}{|c|c|c|c|c|c|c|}
\hline \multirow{2}{*}{$\begin{array}{c}\text { Track } \\
\text { No. }\end{array}$} & \multirow{2}{*}{$\begin{array}{c}\text { View } \\
\text { angle }^{\mathrm{a}}\end{array}$} & \multicolumn{2}{c|}{$\begin{array}{c}\text { Column } \\
\text { (pixel) }\end{array}$} & \multicolumn{2}{c|}{$\begin{array}{c}\text { Row } \\
\text { (pixel) }\end{array}$} \\
\cline { 4 - 7 } & & Mean & Std & Mean & Std \\
\hline \multirow{3}{*}{$\begin{array}{c}\text { Intra- } \\
\text { track }\end{array}$} & \multirow{2}{*}{0236} & $\mathrm{~F}$ & 0.06 & 1.47 & 0.00 & 0.02 \\
\cline { 3 - 7 } & & $\mathrm{B}$ & -0.06 & 1.47 & 0.00 & 0.02 \\
\hline \multirow{3}{*}{$\begin{array}{c}\text { Inter- } \\
\text { track }\end{array}$} & \multirow{2}{*}{0236} & $\mathrm{~F}$ & 1.31 & 0.37 & 0.00 & 0.00 \\
\cline { 3 - 7 } & & $\mathrm{B}$ & -1.31 & 0.37 & 0.00 & 0.00 \\
\cline { 3 - 7 } & & $\mathrm{F}$ & -4.28 & 1.63 & 90.70 & 1.70 \\
\cline { 3 - 7 } & & $\mathrm{B}$ & -4.10 & 1.72 & 91.31 & 1.98 \\
\cline { 3 - 7 } & & $\mathrm{B}$ & 19.11 & 0.52 & -456.92 & 0.92 \\
\hline
\end{tabular}

a "F" and "B" in column "View angle" stand for Forward- and Backward-looking respectively.

Table 2.Back-projection residuals after adjustment

\begin{tabular}{|c|c|c|c|c|c|c|}
\hline & \multirow{2}{*}{$\begin{array}{c}\text { Track } \\
\text { No. }\end{array}$} & \multirow{2}{*}{$\begin{array}{l}\text { View } \\
\text { angle }\end{array}$} & \multicolumn{2}{c|}{$\begin{array}{c}\text { Column } \\
\text { (pixel) }\end{array}$} & \multicolumn{2}{c|}{$\begin{array}{c}\text { Row } \\
\text { (pixel) }\end{array}$} \\
\cline { 3 - 7 } & & & Mean & Std & Mean & Std \\
\hline \multirow{3}{*}{$\begin{array}{c}\text { Intra- } \\
\text { track }\end{array}$} & \multirow{2}{*}{0236} & $\mathrm{~F}$ & 0.02 & 0.36 & 0.00 & 0.02 \\
\cline { 3 - 7 } & & $\mathrm{B}$ & -0.02 & 0.36 & 0.00 & 0.02 \\
\hline \multirow{3}{*}{$\begin{array}{c}\text { Inter- } \\
\text { track }\end{array}$} & \multirow{2}{*}{0236} & $\mathrm{~F}$ & 0.04 & 0.36 & 0.00 & 0.00 \\
\cline { 3 - 7 } & \multirow{2}{*}{0570} & $\mathrm{~B}$ & -0.04 & 0.36 & 0.00 & 0.00 \\
\cline { 3 - 7 } & & $\mathrm{F}$ & -0.14 & 0.26 & -0.15 & 0.15 \\
\cline { 3 - 7 } & & $\mathrm{B}$ & -0.05 & 0.48 & 0.73 & 0.49 \\
\hline
\end{tabular}


Calibration parameters, change of EOPs and ground points are shown in Table 3-Table 6. DOMs before and after adjustment are generated and shown in Figure 3. Compared with the base map from No.0570 track in Figure 3(a), we can see that after adjustment, the mismatch (in Figure 3(b)) between the two DOMs is corrected (in Figure 3(c)).

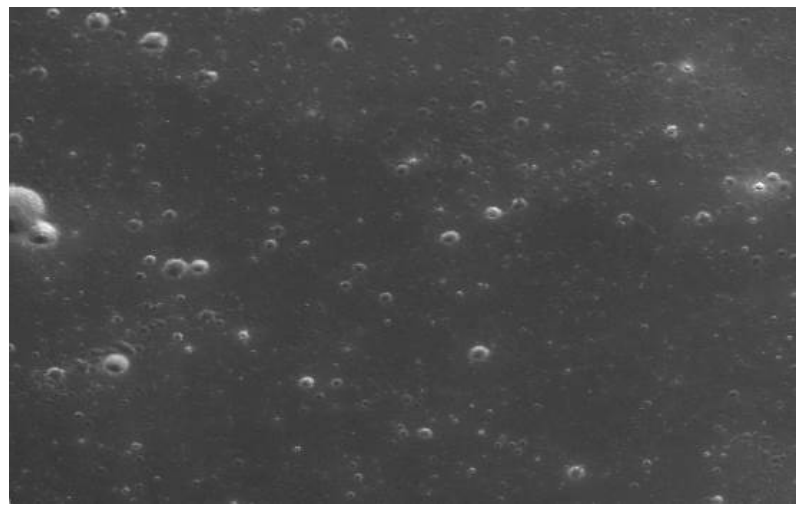

(a)

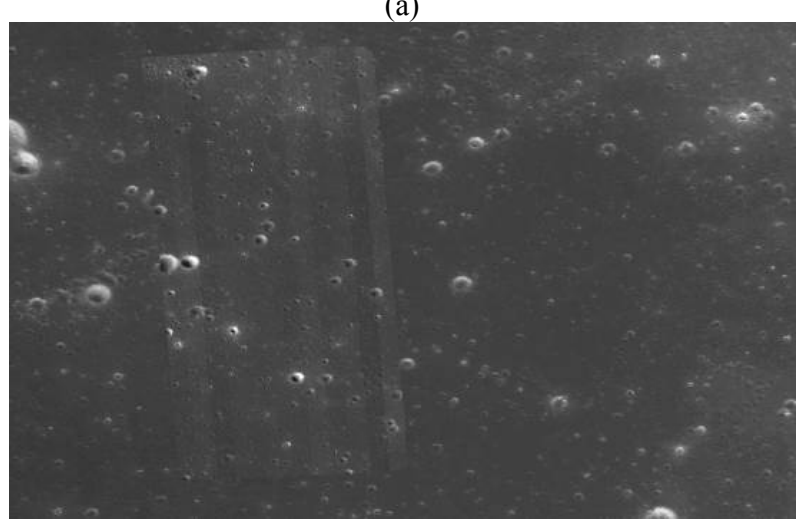

(b)

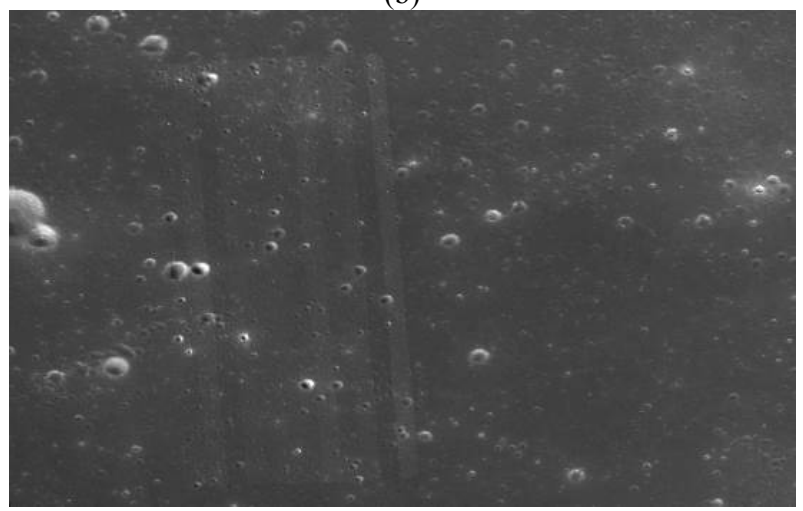

(c)

Figure 3. Overlaid CE-2 DOMs. (a) Base DOM from No.0570 track (7m-resolution), (b) Overlaid DOMs from No.0236 (1.5mresolution) and No.0570 track before adjustment, (c) Overlaid DOMs after adjustment

Table 3.Calibration parameters for CE-2 CCD images

\begin{tabular}{|c|c|c|c|}
\hline View angle & Direction & scale & offset \\
\hline \multirow{2}{*}{$\mathrm{F}$} & $x$ & 1.00000157 & -0.0000797 \\
\cline { 2 - 4 } & $y$ & 1.00001355 & 0.00000609 \\
\hline \multirow{2}{*}{$\mathrm{B}$} & $x$ & 0.99999801 & -0.00004404 \\
\cline { 2 - 4 } & $y$ & 0.99999573 & 0.00002981 \\
\hline
\end{tabular}

Table 4.Change of ground points after adjustment

\begin{tabular}{|c|c|c|c|c|c|c|}
\hline & \multicolumn{2}{|c|}{$X(\mathrm{~m})$} & \multicolumn{2}{c|}{$Y(\mathrm{~m})$} & \multicolumn{2}{c|}{$Z(\mathrm{~m})$} \\
\cline { 2 - 7 } & Mean & Std & Mean & Std & Mean & Std \\
\hline $\begin{array}{c}\text { Intra- } \\
\text { track }\end{array}$ & 2.62 & 5.93 & 3.48 & 11.78 & -3.78 & 6.71 \\
\hline $\begin{array}{c}\text { Inter- } \\
\text { track }\end{array}$ & 2.56 & 6.51 & 0.24 & 2.82 & -1.12 & 6.37 \\
\hline
\end{tabular}

Table 5.Change of position elements of exterior orientation

\begin{tabular}{|c|c|c|c|c|c|c|}
\hline Track & \multicolumn{2}{|c|}{$X s(\mathrm{~m})$} & \multicolumn{2}{c|}{$Y s(\mathrm{~m})$} & \multicolumn{2}{c|}{$Z s(\mathrm{~m})$} \\
\cline { 2 - 7 } No. & Mean & Std & Mean & Std & Mean & Std \\
\hline 0236 & 35.27 & 12.43 & 45.23 & 18.23 & 18.62 & 21.79 \\
\hline 0570 & 174.57 & 48.64 & -46.73 & 37.86 & -58.80 & 67.49 \\
\hline
\end{tabular}

Table 6.Change of pointing elements of exterior orientation

\begin{tabular}{|c|c|c|c|c|c|c|}
\hline Track & \multicolumn{2}{|c|}{$\varphi\left({ }^{\prime}\right)$} & \multicolumn{2}{c|}{$\omega\left({ }^{\prime \prime}\right)$} & \multicolumn{2}{c|}{$\kappa(")$} \\
\cline { 2 - 7 } No. & Mean & Std & Mean & Std & Mean & Std \\
\hline 0236 & -5.82 & 3.35 & 7.55 & 4.47 & 0.14 & 1.07 \\
\hline 0570 & 0.17 & 0.39 & 0.96 & 0.37 & -0.45 & 0.25 \\
\hline
\end{tabular}

\section{GEOMETRIC MODEING OF CE-3 DESENT IMAGES}

CE-3 began to descent from the lunar orbit at an altitude of around $15 \mathrm{~km}$, and when it was about $2 \mathrm{~km}$ above the lunar surface, the descent camera fixed at the bottom of CE- 3 started to take images. During the descending phase, hovering and obstacle avoidance and landing phase (see Figure 4), CE-3 descent camera acquired totally 4,672 images with a resolution higher than $1 \mathrm{~m}$ within an area of $1 * 1 \mathrm{~km}$ and as high as $0.1 \mathrm{~m}$ within a range of $50 \mathrm{~m}$ from the landing point (Liu et al. 2014). Main technical parameters of CE-3 descent camera are listed in Table 7.

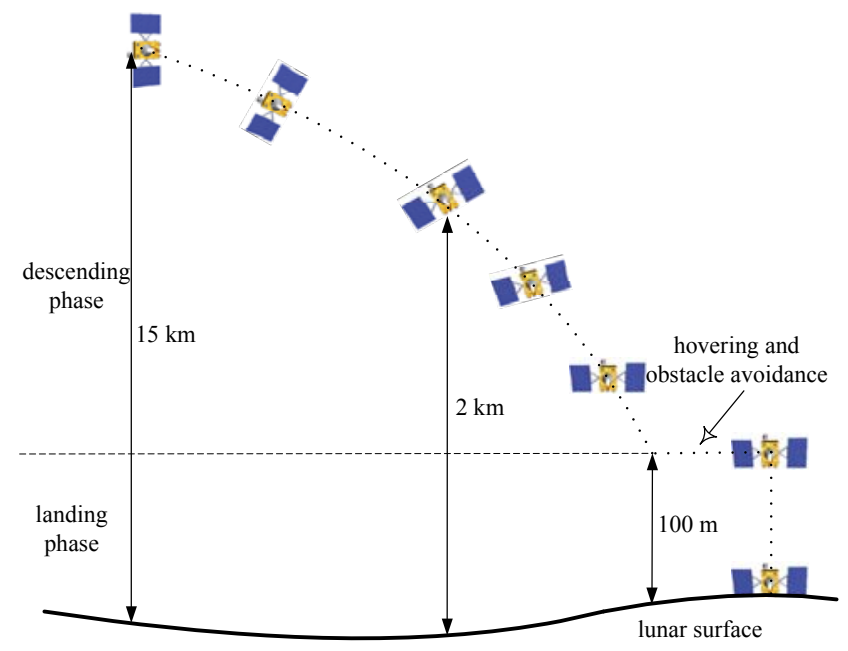

Figure 4. Imaging process of CE-3 descent camera

Table 7.Technical parameters of CE-3 descent camera

\begin{tabular}{|l|l|}
\hline Image size & $1024 * 1024$ pixels \\
\hline Actual imaging distance & $4 \mathrm{~m} \sim 2000 \mathrm{~m}$ \\
\hline Frame frequency & $\geq 10 \mathrm{FPS}$ \\
\hline Acquired Images & 4672 frames \\
\hline Focal length & $8.3 \mathrm{~mm}$ \\
\hline
\end{tabular}




\section{Pixel size}

$$
6.7 \mu \mathrm{m}
$$

180 equally spaced images are selected and incorporated in a self-calibration free network bundle adjustment, and the initial trajectory (including camera positions and attitudes) of the camera is recovered. Then, $26 \mathrm{GCPs}$ are selected from the rectified CE-2 DEM and DOM for absolute orientation. The precision of the GCPs can reach better than $1 \mathrm{~m}$ (Liu et al. 2014) and the DEM and DOM are generated as shown in Figure 5.

Figure 6 shows the rectified DOM from descent images overlaid on DOM from No.0236 track of CE-2. After rectification, the two DOMs match well. Note that there is still a little mismatch at the south edge of the descent DOM, which is because descent images are acquired from south to north, images located in the south are sparse and with low resolution relatively, which affected the accuracy of GCP selection.

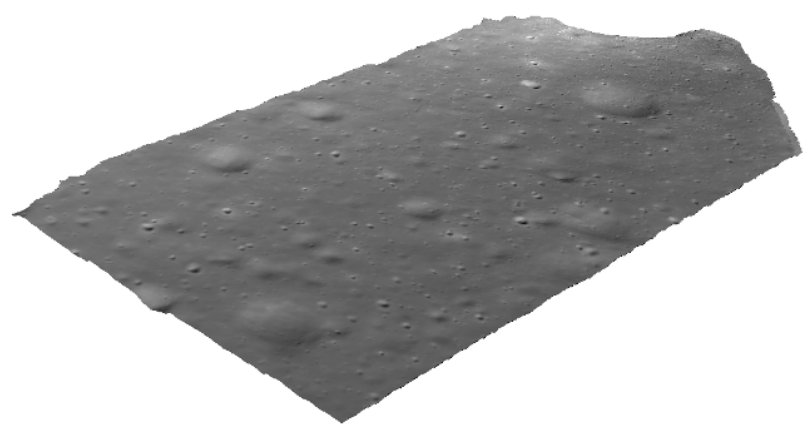

Figure 5. DOM overlaid on DEM generated form CE-3 descent camera (0.4m-resolution)

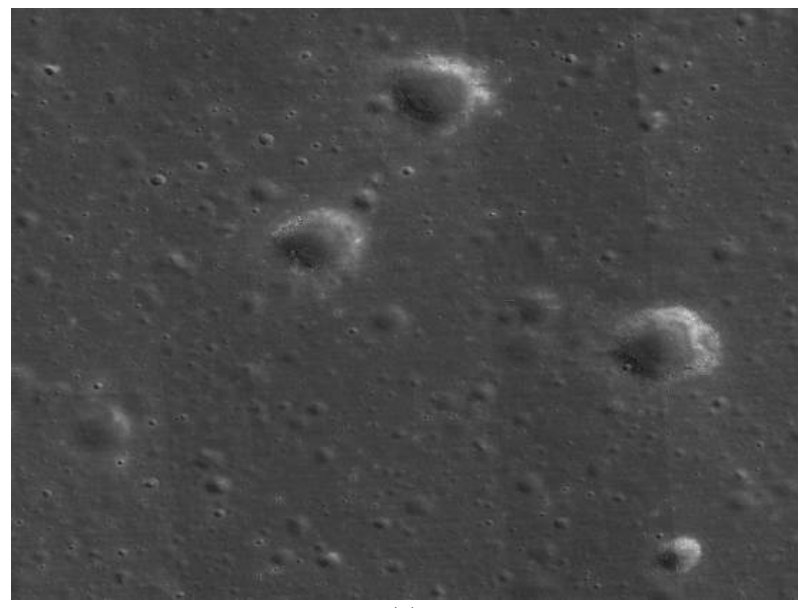

(a)

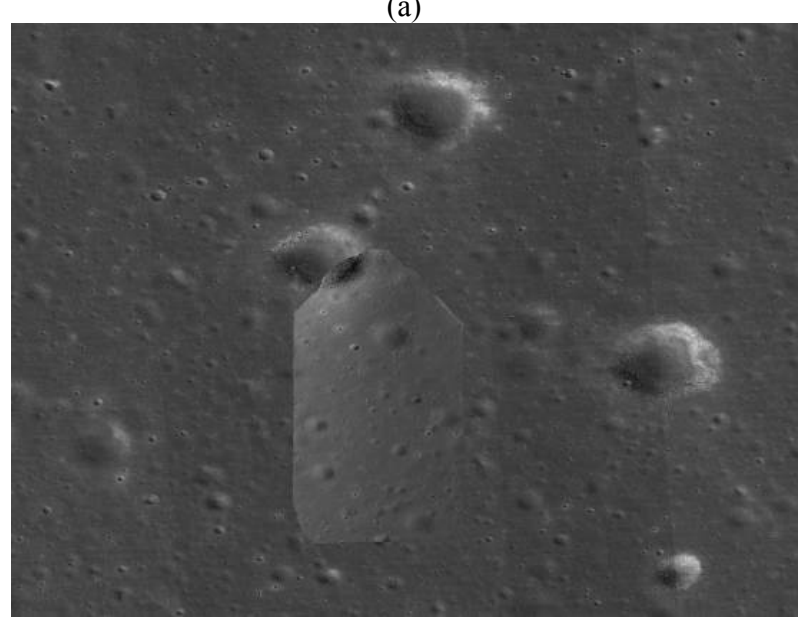

(b)

Figure 6. Overlaid CE-2 and CE-3 DOMs. (a) Base DOM from No.0236 track of CE-2, (b) Overlaid DOMs after bundle adjustment and absolution orientation.

\section{INTEGRATED TOPOGRAPHIC PRODUCT GENERATION}

After CE-2 CCD images, CE-3 descent images are all rectified, topographic data are integrated into form a unified, multi-scale, high precision map of the CE-3 landing site. Figure 7 shows a multi-scale TIN (triangular irregular network) generated using ground points from CE-2 No.0236 track, No.0570 track and CE-3 descent images. It can be seen that at the landing point, the TIN is quite dense for constructing ultra-high resolution topography maps. Figure 8 shows a unified, integrated map at the landing site by overlaying the three DOMs after model refinement and rectification. DOMs are processed by greyscale fusion for better visual effect.

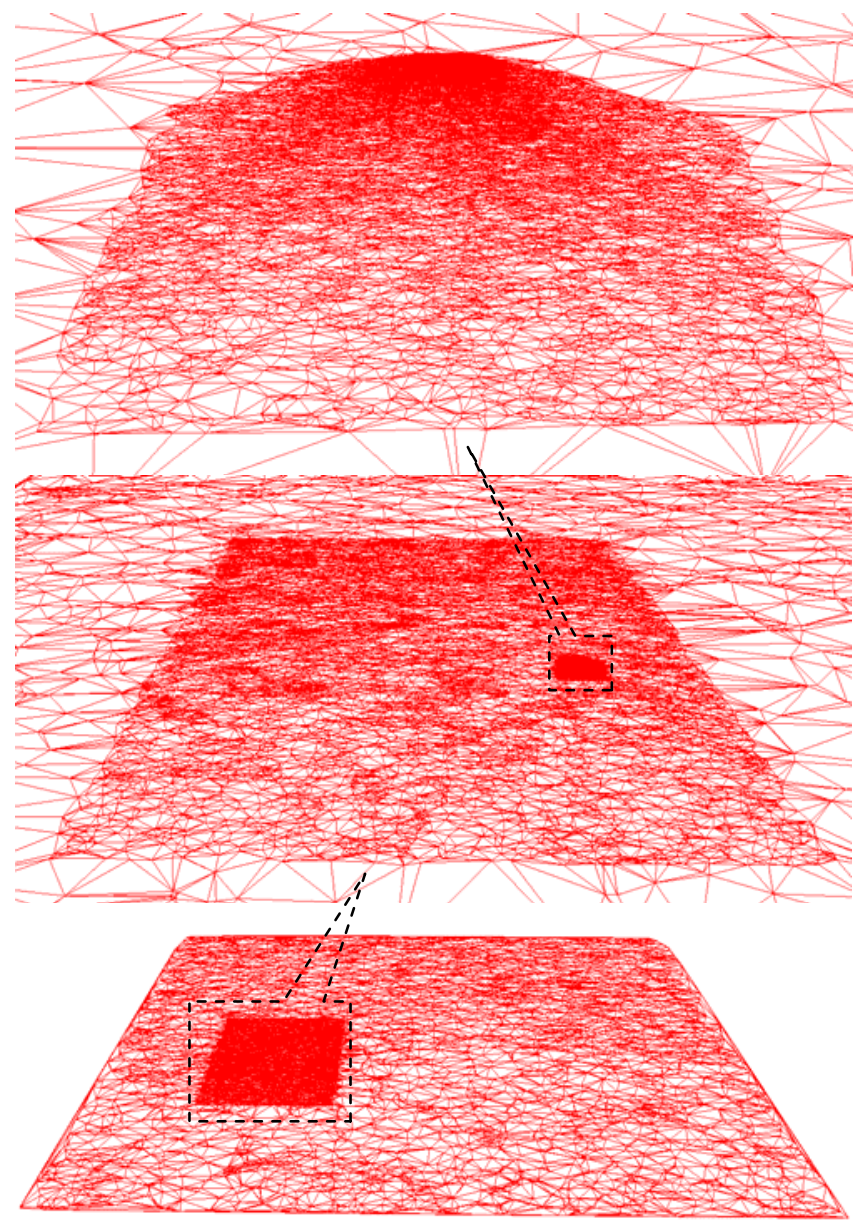

Figure 7. A unified, integrated TIN at the CE-3 landing site. The top, middle and bottom figure show the TINs of descent images, No.0236 and No.0570 images, respectively. 


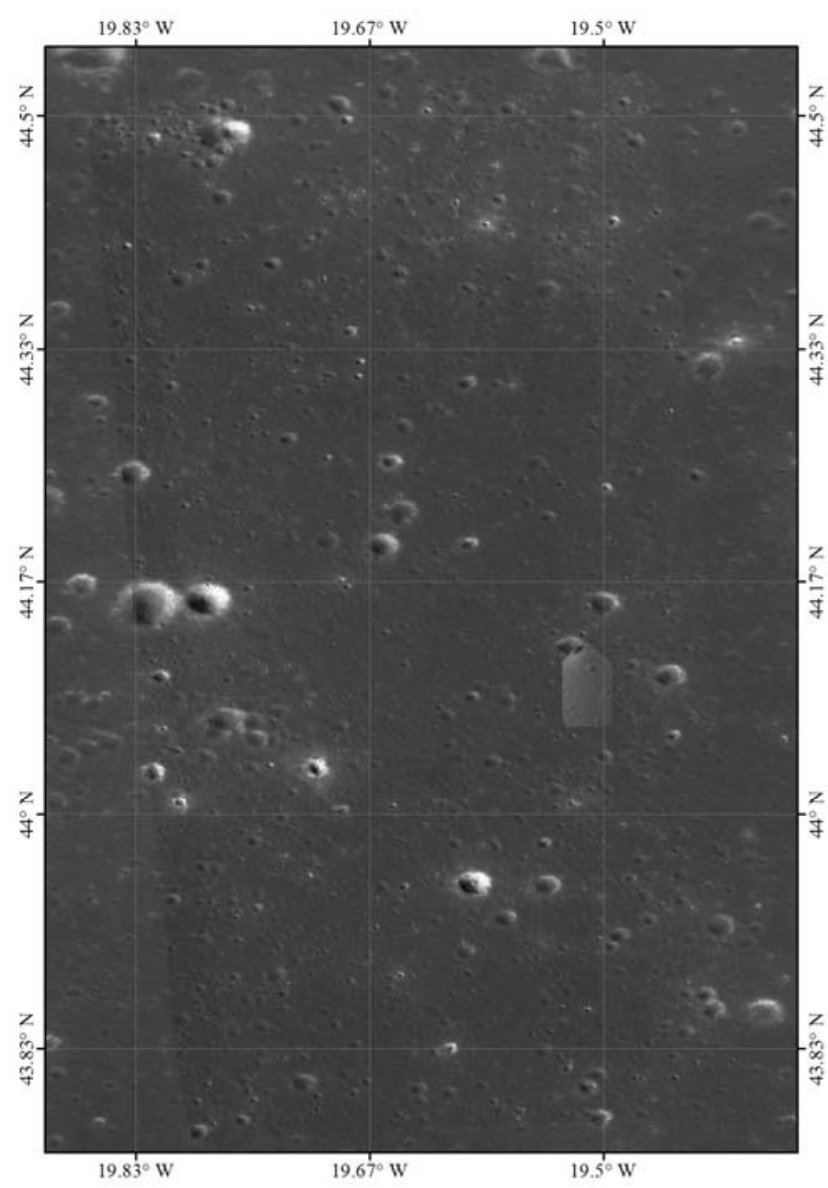

Figure 8. A unified, integrated map at the CE-3 landing site.

\section{REFERENCES}

Di, K., Liu, Y., Liu, B. and Peng, M., 2012. Rigorous photogrammetric processing of Chang'E-1 and Chang'E-2 stereo imagery for lunar topographic mapping. In: The International Achieves of the Photogrammetry, Remote Sensing and Spatial Information Sciences, Melbourne, Australia, Vol. XXXIX, Part B4, pp. 307-312.

Di, K., Liu, Y., Liu, B., Peng, M. and W. Hu., 2014. A selfcalibration bundle adjustment method for photogrammetric processing of Chang'E-2 stereo lunar imagery. IEEE Trans. on Geosci. and Remote Sens., in press.

Li, C., 2012. China publishes the most clear moon map, Defence Science \& Technology Industry, (2), pp. 20-21.

Liu, B., Xu, B., Liu, Z., Liu, Y., Di, K., Tang, G. and Zhou, J., 2014. Descending and landing trajectory recovery of Chang'e 3 lander using descent images. Journal of remote sensing, to be published.

Liu, Y., and K. Di, 2011. Evaluation of rational function model for geometric modeling of Chang'E-1 CCD images. In: The International Achieves of the Photogrammetry, Remote Sensing and Spatial Information Sciences, Guilin China, Vol. XXXVIII, Part 4/W25, pp. 121-125.

Ouyang, Z., 2010. Science results of Chang'e-1 lunar orbiter and mission goals of Chang'e-2. Spacecraft Engineering, 19(5), pp. 1-6.

Wu, B., Hu, H. and Guo, J. , 2014. Integration of Chang'E-2 imagery and LRO laser altimeter data with a combined block adjustment for precision lunar topographic modeling. Earth and Planetary Science Letters, 391, pp. 1-15.

\section{CONCLUSIONS}

In this paper, we presented a method for high precision topographic mapping at Chang'e-3 landing site with multisource data. CE-2 7 m-, 1.5 m-resolution images and CE-3 descent images are used for the mapping with LRO WAC mosaic map and LOLA DTM used as control information. The results proved that self-calibration bundle adjustment and absolute orientation are effective for correcting the inconsistencies among the topographic data generated from different data source. Based on the developed method, unified, multi-scale, high precision topographic products of CE-3 landing site have been generated and used to support surface operations of the CE-3 mission.

\section{ACKNOWLEDGEMENT}

Funding of this research by National Natural Science Foundation of China $(41171355,41301528$ and 41201480) is acknowledged. We thank the Lunar and Deep Space Exploration Science Applications Center of the National Astronomical Observatories (NAOC) and Beijing Aerospace Control Center for providing the CE-2 CCD images, CE-3 descent images and telemetry data. 